\begin{tabular}{|c|l|}
\hline Title & Snapshot imaging Mueller matrix polarimeter using polarization gratings \\
\hline Author(s) & Kudenov, Michael W.; Escuti, Michael J.; Hagen, Nathan; Dereniak, Eustace L.; Oka, Kazuhiko \\
\hline Citation & $\begin{array}{l}\text { Optics Letters, 37(8), 1367-1369 } \\
\text { https:/doi.org/10.1364/0L.37.001367 }\end{array}$ \\
\hline Issue Date & 2012-04_15 \\
\hline Doc URL & http://hdl.handle.net/2115/49323 \\
\hline Rights & ○ 2012 Optical Society of A merica \\
\hline Type & article \\
\hline File Information & OL 37-8_1367-1369.pdf \\
\hline
\end{tabular}

Instructions for use 


\title{
Snapshot imaging Mueller matrix polarimeter using polarization gratings
}

\author{
Michael W. Kudenov, ${ }^{1, *}$ Michael J. Escuti, ${ }^{2}$ Nathan Hagen, ${ }^{3}$ Eustace L. Dereniak, ${ }^{1}$ and Kazuhiko Oka ${ }^{4}$ \\ ${ }^{1}$ College of Optical Science, The University of Arizona, 1630 E. University Boulevard, Tucson, Arizona 85721, USA \\ ${ }^{2}$ Department of Electrical \& Computer Engineering, North Carolina State University, \\ 2410 Campus Shore Drive, Raleigh, North Carolina 27606, USA \\ ${ }^{3}$ Rebellion Photonics, 7457 South Freeway, Houston, Texas 77021, USA \\ ${ }^{4}$ Division of Applied Physics, Hokkaido University, N-13, W-8, Sapporo 060-8628, Japan \\ ${ }^{*}$ Corresponding author: mkudenov@optics.arizona.edu
}

Received December 15, 2011; revised February 24, 2012; accepted February 28, 2012; posted February 29, 2012 (Doc. ID 160054); published April 12, 2012

\begin{abstract}
A snapshot imaging Mueller matrix polarimeter (SIMMP) is theoretically described and empirically demonstrated through simulation. Spatial polarization fringes are localized onto a sample by incorporating polarization gratings (PGs) into a polarization generator module. These fringes modulate the Mueller matrix (MM) components of the sample, which are subsequently isolated with PGs in an analyzer module. The MM components are amplitude modulated onto spatial carrier frequencies which, due to the PGs, maintain high visibility in spectrally broadband illumination. An interference model of the SIMMP is provided, followed by methods of reconstruction and calibration. Lastly, a numerical simulation is used to demonstrate the system's performance in the presence of noise. (c) 2012 Optical Society of America

OCIS codes: $\quad 110.5405,120.5410,260.3160$.
\end{abstract}

Mueller matrix (MM) imaging polarimetry is capable of characterizing the diattenuation, retardation, and depolarization properties of a surface, optical element, or specimen [1]]. As such, it has applications in ground-truth for remote sensing [2], quality control [3], and biomedical imaging [4]]. A sample's MM is often characterized by time-sequentially changing the polarization state of both the generated and analyzed light using rotating polarization elements. By measuring the intensity under at least 16 unique combinations of generated and analyzed polarization states, the 16 Mueller matrix components can be calculated. However, more measurements are often taken to increase the measured signal-to-noise ratio (SNR) and to optimize the condition number of the system's measurement matrix $[4, \underline{5}]$.

Since a conventional MM imaging polarimeter uses temporal scanning, intensity variations caused by sample motion must be minimized and often require an image registration scheme [6]. Misregistration is also caused by systematic errors, such as beam-wander from the rotating optical elements. This further increases the complexity in applications where MM imaging polarimetry may be beneficial, especially for in vivo biomedical imaging of scenes with low inherent contrast [ $\underline{6}]$.

To eliminate the need for image registration and capture all information in a single measurement (i.e., a snapshot), we propose to combine a technique for channeled Mueller matrix spectropolarimetry [7] with an imaging channeled polarimeter based on polarization gratings (PGs) [ㅇ, 9]. The layout of the snapshot imaging Mueller matrix polarimeter (SIMMP) is depicted in Fig. 1. It contains both a generator and analyzer module. In the generator module, the source is either spatially incoherent quasi-monochromatic light or spectrally broadband illumination that is collimated by lens $f_{1}$. Transmission of this light through the linear polarizer $\left(P_{1}\right)$ makes the incident normalized Stokes vector $\mathbf{S}_{\text {in }}=\left(S_{0, \text { in }}(x, y) / 2\right)\left[\begin{array}{llll}1 & 0 & 1 & 0\end{array}\right]^{T}$, where the superscript
$T$ represents the transpose operation and $S_{0, \mathrm{in}}$ is the total incident power. Transmission of this light through PGs $L_{1}$ through $L_{4}$ enables the second re-imaging lens $\left(f_{2}\right)$ to localize polarization interference fringes $[10,11]$ onto the sample. These fringes modulate the Mueller matrix $\mathbf{M}(x, y)$ of the sample by two spatial carrier frequencies, the field of which is then collimated by lens $f_{3}$ into the analyzing optics. It should be mentioned that $\mathbf{M}(x, y)$ is assumed to have no wavelength dependence over the spectral range of the measurement. The 4 beams from the generator are then sheared into 16 beams by the analyzing PGs $L_{5}$ through $L_{8}$. Lastly, the linear polarizer $\left(P_{2}\right)$ enables intensity fringes to be localized onto the focal plane array (FPA) by the re-imaging lens $f_{4}$.

The functional form of the intensity distribution on the FPA was calculated using a combination of Mueller calculus and scalar diffraction theory $[\underline{8}, \underline{12}]$. The irradiance at the FPA, assuming that $f_{1}^{-}=f_{2}=f_{3}=f_{4}$, becomes

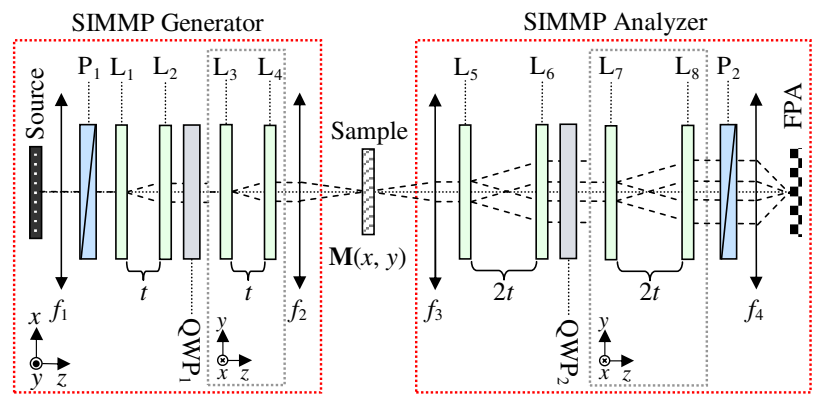

Fig. 1. (Color online) SIMMP optical configuration. PGs $L_{1}, L_{2}$, $L_{5}$ and $L_{6}$ shear the beam along $x$ while $L_{3}, L_{4}, L_{7}$ and $L_{8}$ shear along $y . P_{1}$ and $P_{2}$ are linear polarizers at $45^{\circ}$ while two quarter wave-plates, $\mathrm{QWP}_{1}$ and $\mathrm{QWP}_{2}$, have fast axes oriented at $45^{\circ}$ and $0^{\circ}$, respectively. All PGs have identical grating periods $\Lambda$ and the generator's and analyzer's PGs are separated by a distance $t$ and $2 t$, respectively. 


$$
\begin{aligned}
I(x, y)= & \left|A_{1}\right| \cos \left[\kappa \alpha(x+3 y)+A_{1}^{a}\right] \\
& +\left|A_{2}\right| \cos \left[\kappa \alpha(3 x-3 y)+A_{2}^{a}\right] \\
& +\left|A_{3}\right| \cos \left[\kappa \alpha(x+y)+A_{3}^{a}\right] \\
& +\left|A_{4}\right| \cos \left[\kappa \alpha(3 x-y)+A_{4}^{a}\right] \\
& +\left|A_{5}\right| \cos \left[\kappa \alpha(x-y)+A_{5}^{a}\right] \\
& +\left|A_{6}\right| \cos \left[\kappa \alpha(3 x+y)+A_{6}^{a}\right] \\
& +\left|A_{7}\right| \cos \left[\kappa \alpha(x-3 y)+A_{7}^{a}\right] \\
& +\left|A_{8}\right| \cos \left[\kappa \alpha(3 x+3 y)+A_{8}^{a}\right] \\
& +\left|A_{9}\right| \cos \left[\kappa \alpha(x+2 y)+A_{9}^{a}\right] \\
& +\left|A_{10}\right| \cos \left[\kappa \alpha(3 x-2 y)+A_{10}^{a}\right] \\
& +\left|A_{11}\right| \cos \left[\kappa \alpha(x-2 y)+A_{11}^{a}\right] \\
& +\left|A_{12}\right| \cos \left[\kappa \alpha(3 x+2 y)+A_{12}^{a}\right] \\
& +\left|A_{13}\right| \cos \left[\kappa \alpha(2 x-2 y)+A_{13}^{a}\right] \\
& +\left|A_{14}\right| \cos \left[\kappa \alpha(2 x+2 y)+A_{14}^{a}\right] \\
& +\left|A_{15}\right| \cos \left[\kappa \alpha(x)+A_{15}^{a}\right] \\
& +\left|A_{16}\right| \cos \left[\kappa \alpha(2 y)+A_{16}^{a}\right]+A_{17},
\end{aligned}
$$

where $\kappa=2 \pi / \lambda f_{4}$ is the phase constant, $\lambda$ is the freespace wavelength, and the generator's and analyzer's shear are $\alpha=2 t \lambda / \Lambda$ and $\beta=4 t \lambda / \Lambda$, respectively, where $\Lambda$ is the PG's period. Note that $\kappa \alpha$ and $\kappa \beta$ are constant with wavelength meaning that the carrier frequencies are independent of the illumination's temporal coherence length. This is directly analogous to [] in that the shear depends linearly on the wavelength. Meanwhile, the complex coefficients $A_{k}(x, y)$ represent summations of Mueller matrix elements while $A_{k}^{a}=\arg \left[A_{k}(x, y)\right]$. The $A_{k}$ coefficients are shown in Table $\underline{1}$ and implicitly depend on $x$ and $y$ for clarity.

Table 1. Coefficient Definitions for the Intensity Pattern

\begin{tabular}{ll}
\hline$A_{k}=$ & Coefficient $\times\left(S_{0, \text { in }}(x, y) / 16\right)$ \\
\hline$A_{1}=$ & $-m_{23}-m_{32}-i m_{22}+i m_{33}+2 m_{42}+2 i m_{43}$ \\
$A_{2}=$ & $-m_{23}+m_{32}+i m_{22}+i m_{33}$ \\
$A_{3}=$ & $\quad-m_{23}+m_{32}+i m_{22}+i m_{33}-2 m_{42}$ \\
$A_{4}=$ & $\quad-2 i m_{43}-4 m_{13}+4 i m_{12}$ \\
$A_{5}=$ & $-m_{23}-m_{32}-i m_{22}+i m_{33}$ \\
& $\quad+2 i m_{43}-4 m_{22}+i m_{33}+2 m_{42}+4 m_{12}$ \\
$A_{6}=$ & $-m_{23}+m_{32}+i m_{22}+i m_{33}$ \\
$A_{7}=$ & $-m_{23}+m_{32}+i m_{22}+i m_{33}+2 m_{42}+2 i m_{43}$ \\
$A_{8}=$ & $-m_{23}-m_{32}-i m_{22}+i m_{33}$ \\
$A_{9}=$ & $-2 m_{34}-2 i m_{24}-4 m_{44}$ \\
$A_{10}=$ & $-2 m_{34}+2 i m_{24}$ \\
$A_{11}=$ & $-2 m_{34}-2 i m_{24}+4 m_{44}$ \\
$A_{12}=$ & $2 m_{34}+2 i m_{24}$ \\
$A_{13}=$ & $4 m_{21}-4 i m_{31}$ \\
$A_{14}=$ & $4 m_{21}-4 i m_{31}$ \\
$A_{15}=$ & $8 i m_{14}$ \\
$A_{16}=$ & $8 i m_{41}$ \\
$A_{17}=$ & $8 m_{11}$ \\
\hline
\end{tabular}

In Table 1 , the MM elements are represented as $m_{i j}$, where the subscripts $i$ and $j$ represent the row and column of the $4 \times 4$ element Mueller matrix M. All MM elements are implicitly dependent on $x$ and $y$ for clarity. Fourier transformation of $I(x, y)$ produces 33 channels in $I^{\prime}(\xi, \eta)$, the Fourier domain representation of the measured irradiance, where each channel is proportional to one of the coefficients in Table 1 . A map of the channels in the Fourier domain is depicted in Fig. 2. Extraction of these channels is identical to $[8,12-14]$, in which a 2-dimensional filter is placed at each channel to isolate it.

Once a channel is isolated, its content is inverse Fourier transformed to produce $k$ filtered channels, $C_{k}$, that are proportional to $A_{k}$ modulated by a complex exponential phase. To demodulate the phase from $A_{k}$, each channel's phase is quantified using a previously recorded reference taken of one or more known Mueller matrices $\mathbf{M}_{r}$. From $\mathbf{M}_{r}$, the reference light's $A_{k}$ coefficients $\left(A_{r k}\right)$ are calculated. The $k$ th channel is demodulated and calibrated by

$$
C_{k, \mathrm{cal}}=A_{r k}\left(C_{s k} / C_{r k}\right)\left(C_{r 17} / C_{s 17}\right),
$$

where $C_{s k}, C_{r k}$ are the $k$ th sample and reference channels, respectively, and $C_{r 17}, C_{s 17}$ are included to remove any illumination dependencies in $S_{0 \text {,in }}$ between the reference and sample. Once demodulated, the individually calibrated channels must be algebraically combined to isolate the MM components per Table 2 .

The intensity pattern of Eq. (1) was simulated assuming a spatially uniform input where all $m_{i j}=0.25$. A Poisson-noise-limited $1024 \times 1024$ pixel array was simulated for use as the imaging sensor. The noise was modeled using laboratory measurements taken from an 8-bit Imaging Source 41BU02 machine vision camera at room temperature (gain $=456$, exposure $=1 / 83 \mathrm{~s}$ ). The system's simulated parameters are $t=5.1 \mathrm{~mm}, f_{1}=f_{2}=$ $f_{3}=f_{4}=50 \mathrm{~mm}, \lambda=0.45-0.65 \mu \mathrm{m}$, and $\Lambda=8 \mu \mathrm{m}$. These parameters enable a cutoff frequency, for each channel, of 10.3 cycles $/ \mathrm{mm}$. Sample measurements involved no time-averaging while simulated calibration images were averaged over 20 frames. To ensure a non-zero output within each channel, calibration data were taken assuming two reference targets: (1) a linear polarizer at 0 degrees followed by a quarter-wave retarder at 22.5 degrees (R1) and (2) a quarter-wave retarder

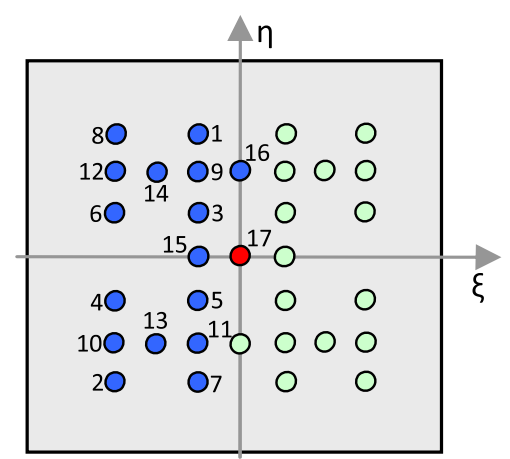

Fig. 2. (Color online) Fourier domain of a channeled image obtained from the SIMMP. Channel numbers correspond to the $k$ subscripts of the $A_{k}$ coefficients per Table $\underline{1}$. Only the non-conjugated channels are numbered for clarity. 
Table 2. Mueller Matrix Solutions from the Fourier domain

\begin{tabular}{|c|c|}
\hline$m_{i j}$ & Channel Combinations $\times\left|C_{r 17}\right| /\left|C_{s 17}\right|$ \\
\hline$m_{11}$ & $\left|C_{s 17}\right| /\left|C_{r 17}\right|$ \\
\hline$m_{12}$ & $\begin{array}{c}-2 \operatorname{Im}\left[A_{r 2} C_{s 2} / C_{r 2}-A_{r 3} C_{s 3} / C_{r 3}-A_{r 5} C_{s 5} / C_{r 5}\right. \\
\left.+A_{r 8} C_{s 8} / C_{r 8}\right]\end{array}$ \\
\hline$m_{13}$ & $\begin{array}{c}-2 \operatorname{Re}\left[A_{r 1} C_{s 1} / C_{r 1}+A_{r 3} C_{s 3} / C_{r 3}+A_{r 5} C_{s 5} / C_{r 5}\right. \\
\left.+A_{r 7} C_{s 7} / C_{r 7}\right]-m_{23} / 2\end{array}$ \\
\hline$m_{14}$ & $-2 \operatorname{Im}\left[A_{r 15} C_{s 15} / C_{r 15}\right]$ \\
\hline$m_{21}$ & $4 \operatorname{Re}\left[A_{r 13} C_{s 13} / C_{r 13}+A_{r 14} C_{s 14} / C_{r 14}\right]$ \\
\hline$m_{22}$ & $\begin{array}{c}4 \operatorname{Im}\left[A_{r 2} C_{s 2} / C_{r 2}-A_{r 4} C_{s 4} / C_{r 4}+A_{r 6} C_{s 6} / C_{r 6}\right. \\
\left.-A_{r 8} C_{s 8} / C_{r 8}\right]\end{array}$ \\
\hline$m_{23}$ & $\begin{array}{c}-4 \operatorname{Re}\left[A_{r 2} C_{s 2} / C_{r 2}+A_{r 4} C_{s 4} / C_{r 4}+A_{r 6} C_{s 6} / C_{r 6}\right. \\
\left.+A_{r 8} C_{s 8} / C_{r 8}\right]\end{array}$ \\
\hline$m_{24}$ & $-4 \operatorname{Im}\left[A_{r 9} C_{s 9} / C_{r 9}+A_{r 11} C_{s 11} / C_{r 11}\right]$ \\
\hline$m_{31}$ & $-2 \operatorname{Im}\left[A_{r 13} C_{s 13} / C_{r 13}+A_{r 14} C_{s 14} / C_{r 14}\right]$ \\
\hline$m_{32}$ & $\begin{array}{c}-4 \operatorname{Re}\left[A_{r 1} C_{s 1} / C_{r 1}-A_{r 3} C_{s 3} / C_{r 3}+A_{r 5} C_{s 5} / C_{r 5}\right. \\
\left.-A_{r 7} C_{s 7} / C_{r 7}\right]\end{array}$ \\
\hline$m_{33}$ & $\begin{array}{c}4 \operatorname{Im}\left[A_{r 2} C_{s 2} / C_{r 2}+A_{r 4} C_{s 4} / C_{r 4}+A_{r 6} C_{s 6} / C_{r 6}\right. \\
\left.+A_{r 8} C_{s 8} / C_{r 8}\right]\end{array}$ \\
\hline$m_{34}$ & $4 \operatorname{Re}\left[A_{r 10} C_{s 10} / C_{r 10}+A_{r 12} C_{s 12} / C_{r 12}\right]$ \\
\hline$m_{41}$ & $2 \operatorname{Im}\left[A_{r 16} C_{s 16} / C_{r 16}\right]$ \\
\hline$m_{42}$ & $\begin{array}{c}4 \operatorname{Re}\left[A_{r 1} C_{s 1} / C_{r 1}-A_{r 4} C_{s 4} / C_{r 4}-A_{r 6} C_{s 6} / C_{r 6}\right. \\
\left.+A_{r 7} C_{s 7} / C_{r 7}\right]\end{array}$ \\
\hline$m_{43}$ & $\begin{array}{c}-4 \operatorname{Im}\left[A_{r 1} C_{s 1} / C_{r 1}-A_{r 4} C_{s 4} / C_{r 4}-A_{r 6} C_{s 6} / C_{r 6}\right. \\
\left.+A_{r 7} C_{s 7} / C_{r 7}\right]\end{array}$ \\
\hline$m_{44}$ & $-2 \operatorname{Re}\left[A_{r 9} C_{s 9} / C_{r 9}-A_{r 11} C_{s 11} / C_{r 11}\right]$ \\
\hline
\end{tabular}

at 22.5 degrees followed by a linear polarizer at 0 degrees (R2). Here, reference R1 is used to calibrate channels 1-15 and 17 while R2 is used to calibrate channel 16 .

First, the MTF was quantified by calculating the contrast of spatial frequencies spanning 0.1 to 10.3 cycles $/ \mathrm{mm}$. The MTF is provided in Fig. 3(a) and is primarily influenced by the shape of a band-limiting window that was used to prevent cross talk between adjacent channels. Such band-limiting can be physically realized by defocusing the sample while maintaining focus on the fringe localization field from the generator [8]. However, for the purposes of this simulation we used a Hamming window with a full-width at half-maximum frequency of 5.4 cycles $/ \mathrm{mm}$.

Each reconstructed MM element's SNR was also calculated and is depicted in Fig. 3(b). Generally, MM components that occupy their own channel (e.g., $m_{14}$ and $m_{41}$ ) have larger SNRs than MM components in highly multiplexed channels (e.g., $m_{22}, m_{23}, m_{33}, m_{42}$ and $m_{43}$ ).

The spatial output of a quarter-wave vortex retarder, on a $4.8 \times 4.8 \mathrm{~mm}$ square substrate, was also simulated [15]. A depiction of the ideal Mueller matrix and the

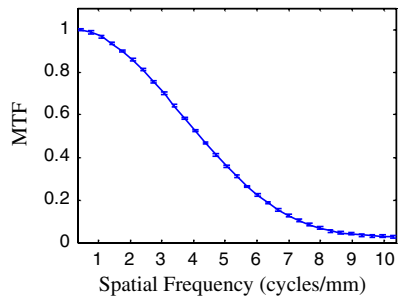

(a)

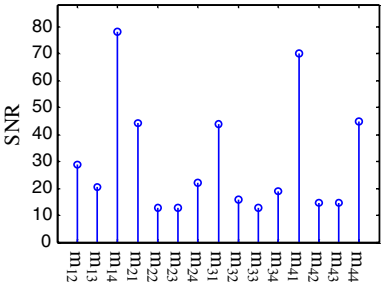

(b)
Fig. 3. (Color online) (a) Average MTF response of all 17 channels where error bars represent one standard deviation, (b) the SNR of each Mueller matrix element.

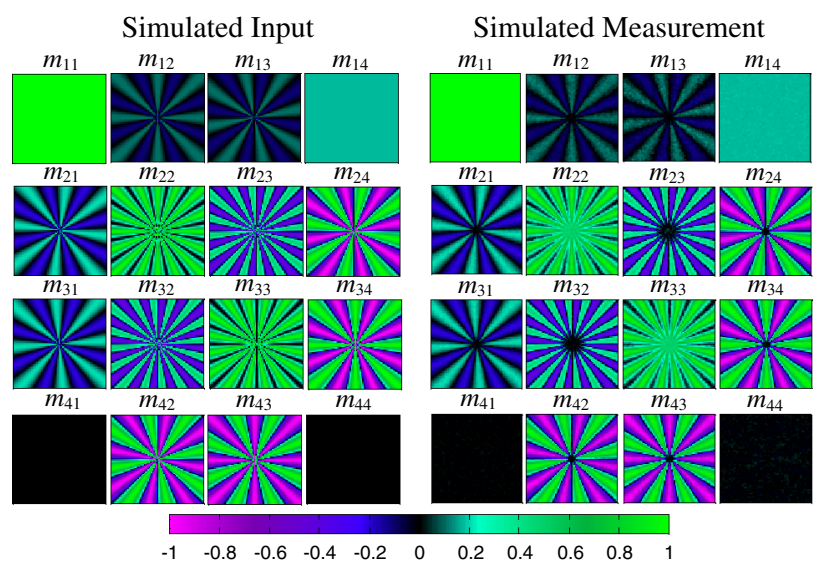

Fig. 4. (Color online) Simulated input (left) and measured (right) Mueller matrix of the quarter-wave vortex retarder.

simulated output are provided in Figs. 4(a) and 4(b), respectively. From the simulated measured data, it is clear that spatial frequencies near the center of the vortex are not discernable, while lower spatial frequency information is preserved. This relates to the spatial band limiting discussed previously.

The SIMMP approach takes advantage of the rapid advance in detector array technology, allowing users to use large pixel count arrays in order to achieve fast readout rates. This allows a 16 -fold improvement in measurement speed at the cost of a 7-fold reduction in image resolution (in each $x$ and $y$ direction). Such advantages may be beneficial in biomedical imaging applications for real-time diagnostics or for imaging a large number of samples in rapid succession for quality control applications. Use of PGs offers a lower cost, a more compact size, and larger apertures over birefringent crystal optics with the capability of using spectrally broadband illumination.

\section{References}

1. D. H. Goldstein, Polarized Light (CRC, 2011).

2. B. D. Bartlett, M. G. Gartley, D. W. Messinger, C. Salvaggio, and J. R. Schott, J. Appl. Remote Sens. 4, 043552 (2010).

3. F. Delplancke, Appl. Opt. 36, 5388 (1997).

4. K. M. Twietmeyer, R. A. Chipman, A. E. Elsner, Y. Zhao, and D. VanNesdale, Opt. Express 16, 21339 (2008).

5. S. Murali, "Analysis of an interferometric Stokes imaging polarimeter," Ph. D. dissertation (University of Arizona, 2010).

6. S. Guyot, M. Anastasiadou, E. Delechelle, and A. D. Martino, Opt. Express 157393 (2007).

7. N. Hagen, K. Oka, and E. L. Dereniak, Opt. Lett. 32, 2100 (2007).

8. M. W. Kudenov, M. J. Escuti, E. L. Dereniak, and K. Oka, Appl. Opt. 50, 2283 (2011).

9. C. Oh and M. J. Escuti, Opt. Lett. 33, 2287 (2008).

10. S. Pancharatnam, Proc. Indian Acad. Sci. A 44, 247 (1956).

11. M. Mujat, A. Dogariu, and E. Wolf, J. Opt. Soc. Am. A 21, 2414 (2004).

12. K. Oka and N. Saito, Proc. SPIE 6295, 629508 (2006).

13. K. Oka and T. Kaneko, Opt. Express 11, 1510 (2003).

14. M. W. Kudenov, M. E. L. Jungwirth, E. L. Dereniak, and G. R. Gerhart, Opt. Express 17, 22520 (2009).

15. S. C. McEldowney, D. M. Shemo, R. A. Chipman, and P. K. Smith, Opt. Lett. 33, 134 (2008). 